\title{
¿NO ES LA FORMA? LA CONTRIBUCIÓN POLÍTICO-CULTURAL DE LAS LUCHAS ESTUDIANTILES A LA EMERGENCIA DEL LARGO OCTUBRE CHILENO ${ }^{1}$
}

\author{
JuAN PABlo Paredes P. ${ }^{2}$ \\ KATIA VALENZUELA FUENTES ${ }^{3}$
}

\begin{abstract}
RESUMEN
El trabajo plantea, a partir de la movilización estudiantil chilena en más de una década, un análisis de su injerencia en la revuelta iniciada en octubre 2019. Sobre la base de materiales de investigaciones previas, se analizan tres coyunturas polémicas: la rebelión pingüina de 2006, el movimiento estudiantil de 2011 y el movimiento feminista de 2018, para definir sus alcances culturales. Se concluye identificando consecuencias culturales en la subjetividad, en los estilos de protesta, en las reglas del sentir y en la economía moral de la ciudadanía, que generaron condiciones político-culturales favorables para la coyuntura crítica de octubre.
\end{abstract}

PALABRAS CLAVE: ANÁLISIS CULTURAL, PROTESTAS ESTUDIANTILES, COYUNTURAS CRÍTICAS.

1 El trabajo agradece el financiamiento de la Agencia Nacional de Investigación y Desarrollo de Chile (ANID), mediante los proyectos, FONDECYT-Postdoctorado 3170504 y CONICYT-REDI 170473.

2 Dr. en Ciencias Sociales. Académico-Investigador, Departamento de Ciencias Sociales, Facultad de Ciencias Sociales y Economía, Universidad Católica del Maule, Talca, Chile. Correo electrónico: jparedes@ucm.cl

3 Dra. en Ciencias Políticas. Académica Departamento de Planificación Territorial, Facultad de Ciencias Ambientales, Universidad de Concepción. Correo electrónico: kavalenzuela@udec.cl 


\title{
NÃO É A FORMA? A CONTRIBUIÇÃO POLÍTICA E CULTURAL DAS LUTAS ESTUDANTIS PARA A EMERGÊNCIA DO LONGO OUTUBRO CHILENO
}

\begin{abstract}
RESUMO
O trabalho propõe, a partir da mobilização estudantil chilena em mais de uma década, uma análise de sua ingerência na revolta iniciada em outubro de 2019. Com base em materiais de pesquisas anteriores, são analisadas três conjunturas polêmicas: a revolta dos pinguins de 2006, o movimento estudantil de 2011 e o movimento feminista de 2018, para definir suas abrangências culturais. Como conclusão, são identificadas consequências culturais na subjetividade, nos estilos de protesto, nas regras do sentir e na economia moral dos cidadãos, que geraram condições políticas e culturais favoráveis para a conjuntura crítica de outubro.
\end{abstract}

PALAVRAS-CHAVE: ANÁLISE CULTURAL, PROTESTOS ESTUDANTIS, CONJUNTURAS CRÍTICAS.

\section{ISN'T IT THE WAY? THE POLITICAL-CULTURAL CONTRIBUTION OF STUDENT STRUGGLES TO THE EMERGENCE OF THE LONG CHILEAN OCTOBER}

\begin{abstract}
The work brings up an analysis of the relevance of Chilean student demonstrations throughout more than a decade for the social outbreak started last October 2019. On the basis of material from previous research, three controversial junctures are analyzed (Penguin Revolution in 2006, the student movement in 2011 and the feminist movement in 2018) in order to measure their cultural reach. As a conclusion, cultural consequences are identified in subjectivity, protest styles, rules to feel and moral economy of citizens, all of them generating favorable political and cultural conditions for the critical junctures in October.
\end{abstract}

KEYWORDS: CULTURAL ANALYSIS, STUDENT PROTESTS, CRITICAL JUNCTURES. 


\section{INTRODUCCIÓN}

La dictadura cívico-militar chilena (1973-1989) realizó una serie de transformaciones estructurales fundadas en una «revolución capitalista» (Gárate, 2012), específicamente neoliberal, cuyos ejes fueron la privatización de lo público y la mercantilización de lo social, donde el Estado asume un rol subsidiario, pero con una participación activa en la configuración de los mercados especializados (Gárate, 2012; Undurraga, 2014; Madariaga, 2019). La operación de instauración político-jurídica del neoliberalismo se dio con la Constitución de 1980, fundando una estructura sociopolítica y económica en el país (Heiss, 2020), que redefine la relación entre política, sociedad y mercado.

En educación, la modernización neoliberal implicó transitar hacia un modelo descentralizado y dominado por la lógica mercantil. El Estado asume un rol regulador y de intervención focalizada, bajo la premisa de que la libertad del mercado y la competencia entre actores incidirían positivamente en la calidad de la educación (Bellei, Cabalín y Orellana, 2014). Con tal foco, las reformas en la educación primaria y secundaria dan un giro hacia el control municipal - la llamada municipalización-, y el Estado aporta vía subsidios según cumplimiento de metas (Assaél et al., 2011). En educación superior se define el principio de autofinanciamiento y el acceso a recursos estatales vía la competencia entre planteles públicos y privados. El Estado entrega financiamiento básico directo a los planteles estatales e indirectos a todo plantel, independiente de su naturaleza, mediante el mecanismo de captura de los mejores alumnos en una lógica de competencia. Ambas políticas se orientan a fomentar las ideas de libertad al interior del mercado, libertad de enseñanza y libertad de elección de su educación por parte de los individuos y sus familias (Assaél et al., 2011), definiendo la libertad al centro de la educación, por sobre un derecho social garantizado.

Con el regreso a la democracia, se realizaron algunas modificaciones, aunque la lógica del mercado educativo no fue transformada sino más bien regulada (Assaél et al., 2011). Ejemplo de lo anterior es la creación del Crédito con Aval del Estado (CAE) con el respaldo de la banca, el año 2005, para mejorar el acceso a la educación, que generó resultados perversos como el alto y masivo endeudamiento. Lo público siguió restringido a la tarea de coordinación y vigilancia por parte de las instituciones estatales, en un sistema fundamentalmente constituido por actores privados (Bellei, 2015; Atria, 2014). 
Aunque los gobiernos democráticos reformaron el modelo social impuesto en la dictadura (Madariaga, 2019), definieron una institucionalidad elitista y tecnocrática, con baja participación ciudadana, que derivó en despolitización y atomización vía un proceso de creciente descolectivización (Báez, 2017; Silva, 2009), esto es, una democracia de baja intensidad (Rovira, 2007). Los espacios de socialización fueron preferentemente los centros comerciales y de servicios, con una ciudadanía definida por su participación en el mercado y el consumo, produciendo un tipo de subjetividad pasiva y orientada al consumo, que fue refrendada en los espacios educativos mercantilizados (Orellana et al., 2019; Paredes, 2019). Por otro lado, el efecto social del modelo neoliberal fue la desigualdad y segregación en gran parte de la población (PNUD, 2016).

Este es parte del escenario de injusticia que se cuestionó desde octubre 2019, bajo la idea de estallido social. Si bien la etiqueta es apropiada por su carácter acontecimental, no da cuenta de las luchas sociales y procesos colectivos anteriores que se cultivaron en el Chile postdictadura, como las estudiantiles (Paredes, 2019), poblacionales (Angelcos y Peréz, 2017), socioterritoriales (Delamaza, et al., 2017), del mundo del trabajo (Julian, 2018) y las pensiones (Rozas y Maillet, 2018), feministas (Lamadrid y Navarrete, 2019) e indígenas (Pineda, 2012). Aunque varios son los conflictos que nutren el octubre chileno de 2019, fueron estudiantes secundarios los que prendieron la mecha, con su acto de evasión y su llamado a la desobediencia civil contra el alza del pasaje del metro los días previos al 18 de octubre (18-O). Tal presencia habilita preguntar por la contribución estudiantil a las condiciones que posibilitaron el acontecimiento del 18-O, como su permanencia. Específicamente, ¿cómo contribuyeron las protestas estudiantiles, sus prácticas, discursos y demandas de más de una década en la ocurrencia del acontecimiento de octubre?

El supuesto guía de la indagación señala que la injerencia de la movilización estudiantil en el largo octubre chileno es significativamente cultural, generando condiciones político-culturales para su ocurrencia. Para demostrarlo, presentamos los argumentos de la siguiente manera. A continuación, planteamos la relación entre movilización estudiantil y alcances culturales (1). Seguidamente, formulamos el análisis de coyuntura como alternativa metodológica (2). Posteriormente, analizamos cada período identificado, enfatizando lo cultural en ellos (3, 4 y 5). Finalmente, planteamos una discusión junto a las conclusiones del estudio (6). 
El trabajo pretende aportar a la comprensión del movimiento estudiantil desde sus outcomes culturales, como promotor de un contexto favorable al acontecimiento de octubre 2019.

\section{MOVIMIENTO ESTUDIANTIL CHILENO RECIENTE Y CONSECUENCIAS CULTURALES}

El movimiento estudiantil chileno, desde 2006, ha gozado de vasta producción académica. Se ha prestado atención a sus repertorios (Aguilera, 2012; Tricot, 2012), sus estrategias políticas (Montero et al., 2017), su formulación como problema público (Paredes y Araya, 2020), o su alcance generacional (Santibáñez y Ganter, 2016).

La literatura de los movimientos sociales ha consignado tres ámbitos preferentes para el estudio de sus consecuencias: políticos, biográficos y culturales (Bossi et al., 2016; Giugni, 2008). Para el caso del movimiento estudiantil, los alcances políticos e institucionales de sus protestas, durante los últimos tres lustros, han sido bien documentados (Donoso y Somma, 2019; Montero, 2018; Penaglia, 2019; Somma, 2012), generando mucha evidencia empírica sobre sus alcances y limitaciones. Una variante de la literatura se enfoca en las transformaciones organizacionales del actor estudiantil (Mella et al., 2016; Avendaño, 2014). Algunos estudios ponen el acento en la relevancia del factor ideológico y en los efectos de legitimidad que la movilización produce (Fleet y Guzmán Concha, 2016; Fleet, 2011). Mientras, otra literatura se concentra en la estructura social mediante un análisis de clase (Ruiz, 2015; Labarca, 2016). Estos trabajos privilegian la entrada política, ya sea organizacional, institucional o estructural de los alcances de la movilización.

En contraste, la dimensión cultural ha sido menos desarrollada. En relación con la cultura, pueden encontrarse trabajos con énfasis en la configuración identitaria (Aguilera, 2011) o militante (Paredes, Ortiz y Araya, 2018; Valenzuela, 2015), en la apropiación del espacio público (Paredes, 2018; Berroeta y Sandoval, 2014; Urzúa, 2015), en los discursos (Haitovic et al., 2012), en las prácticas de autonomía (Valenzuela, 2015), en las emociones (Ganter et al., 2017; Montoni, 2019; Paredes y Otárola, 2019), el uso de redes sociales (Cárdenas, 2016; Cabalín, 2014). Si bien estos estudios consideran el factor cultural de las movilizaciones, muy pocos analizan los alcances fuera de la 
movilización, lo que vuelve problemático el estudio de las consecuencias en su dimensión cultural.

Un segundo problema asociado a tal literatura es que no existe acuerdo sobre el lugar ni la definición de lo cultural en cada planteamiento. Algunos utilizan la cultura subordinada a otra clave analítica, ya sea emociones, identidad, prácticas; otros no remiten a la categoría y la reemplazan por los conceptos de representación, discurso, comunicación o subjetividad. De tal forma, se configura una suerte de archipiélagos temáticos que no permiten el intercambio sistémico en el área, menos un diálogo fecundo y propositivo en torno a lo cultural.

Para los fines de este trabajo, entendemos la cultura como una dimensión constitutiva de la vida social — en adelante lo cultural-, que presenta varios alcances para ella. Primero, lo cultural es el proceso de creación, producción, circulación y usos de significados, a través de acciones, interacciones, relaciones e instituciones, siendo una dimensión constitutiva de lo social (Alexander, 2003; Hall, 2016; Spillman, 2020; Williams, 2003). Segundo, tal dimensión remite a expresiones compartidas por individuos, grupos y colectivos, encarnada en valores, normas, creencias, lenguaje, rituales, símbolos, íconos e identificaciones colectivas (Alexander, 2003; Hall, 2016; Swidler, 1986), por ende, es tanto material como simbólica. Tercero, tiene centralidad en la conformación, permanencia y éxito de los movimientos de protestas (Jasper, 1997, 2016), en su dimensión interna, para la creación de un sentido compartido de identidad en los participantes del movimiento (Polleta y Jasper, 2001), en la creación de un sentido de solidaridad, reclutar miembros y consolidar el compromiso (Polletta y Jasper, 2001; Paredes y Otárola, 2019). Como en su dimensión externa, ya sea en la creación de significados con resonancia social y alcance público, logrando incidir en la esfera social y pública (Cefaï, 2011; Paredes y Araya, 2020).

La definición anterior permite ubicar lo cultural al interior de un programa de investigación de alcance empírico con cierta autonomía analítica, respecto de las dimensiones estructurales y agenciales de lo social (Alexander, 2003; Hall, 2016; Williams, 2003). Sin embargo, a diferencia del programa fuerte de sociología cultural de Alexander (2003), que entiende la cultura con una autonomía analítica total, planteamos una autonomía relativa de lo estructural, pues en el estudio de las luchas colectivas y movimientos sociales se requiere de su conexión con las configuraciones de poder y las estructuras políticoideológicas de la sociedad (Grossberg, 2010; Hall, 2016). Proponemos un intercambio entre herramientas del programa fuerte en sociología cultural y los 
estudios culturales en la tradición de Birmingham, tomando partido por los últimos para definir una entrada político-cultural.

En lo relativo a las consecuencias culturales de los movimientos sociales, la literatura certifica que han sido poco estudiadas (Amenta y Polleta, 2019; Earl, 2004; Giuni, 2008; Van Dyke y Taylor, 2018). Inicialmente se limitaron al ámbito de las creencias y valores, prácticas simbólicas, o conformación de identidades culturales o subculturas (Earl, 2004; Giuni, 2008). Actualmente, la investigación ha complejizado el análisis sumando la consideración a los efectos en los contextos culturales (Amenta y Polleta, 2019; Van Dyke y Taylor, 2018). Este último tópico es el que enfatizamos acá.

En el trabajo utilizamos el estudio de lo cultural en tres formatos de expresión, cognitivo, moral y emocional (Jasper, 2106, 1997), que se materializan en los movimientos sociales en formas de actuación, ideas y artefactos (Johnston, 2009; Van Dyke y Taylor, 2019). A través de estos, planteamos algunas de las consecuencias culturales de las movilizaciones estudiantiles, lo que nos lleva a revisar los alcances metodológicos.

\section{Aspectos Metodológicos}

El escrito se formula como un ensayo de investigación (Gusfield, 1981), que combina varias experiencias de investigación en torno a una problemática en común, y se elabora desde una óptica interpretativa de las consecuencias culturales de los movimientos estudiantiles, sustentada en las herramientas de la investigación cualitativa (Cuevas y Paredes, 2012). Tres tipos de fuentes permiten elaborar este trabajo, provenientes de tres investigaciones previas sobre las movilizaciones estudiantiles, una investigación doctoral (2011-2015); una postdoctoral (2017-2019); la tercera, una investigación con financiamiento externo realizada entre los años 2018-2019. Se consideran algunos artefactos de protestas sustentados en un archivo fotográfico propio, obtenido de la observación participante de algunas protestas en las coyunturas 2011 y 2018, especialmente la primera. Segundo, se realizaron cuarenta entrevistas en profundidad a activistas del movimiento estudiantil que participaron de las manifestaciones 2006, 2011 y 2018. Tercero, se utilizó un análisis de prensa de opinión de diarios digitales de circulación nacional, para los tres momentos. Las fuentes empíricas de las entrevistas se utilizan de manera limitada, con la finalidad de reforzar e ilustrar algún punto. 
Con tales materiales se realiza un análisis de coyuntura sobre cada una de las expresiones de movilización estudiantil más relevantes de los últimos quince años, 2006, 2011 y 2018, enfatizando la dimensión cultural para reconstruirlos y proyectar sus alcances para el acontecimiento de octubre de 2019. A partir de las fuentes de información ya mencionadas, en diálogo con literatura, se reconstruyen las coyunturas polémicas fomentadas por la acción contenciosa del movimiento estudiantil en diferentes momentos, entendidas como totalidad epistémica (Grossberg, 2010; Hall, 2016), antes que empíricas. Llamaremos coyunturas polémicas a los contextos marcados por la acción contenciosa del movimiento estudiantil que generan revuelo público y tienen alcances políticos. En tanto unidades epistémicas, se configuran en torno a problemáticas que permiten su reconstrucción como una totalidad (Grossberg, 2010).

Grossberg (2010) reconoce como punto de arranque la tensión entre el modo de estructuración del poder político-económico en la sociedad y las voces sociales que plantean críticas, demandas o se oponen a tal orden. Tales tensiones conforman las problemáticas que posibilitan el análisis coyuntural, de las que Grossberg (2010) identifica la de subjetividad (experiencia), de agencia y resistencia (movilización), de la hegemonía (estructura político-ideológica), la epistemológica (del saber) y la de periodización histórica. El análisis cultural propuesto nos permite establecer relaciones entre cada uno de los planos señalados, aunque nosotros acentuamos solo algunas.

A continuación, presentamos el análisis ordenado en torno a tres coyunturas polémicas que no alcanzan a producir una dislocación del orden, aunque generan un contexto favorable a la movilización social opuesta al modelo hegemónico. Estas son: la pingüina, la de gratuidad y la feminista. Posteriormente, analizamos su influencia para la emergencia y configuración de la coyuntura crítica del largo octubre chileno, que sí produce un momento crítico de distanciamiento político-cultural radical con el modelo sociopolítico existente.

\section{La Coyuntura Pingüina (2006)}

El 2006, la movilización de los estudiantes secundarios durante el primer gobierno de Michelle Bachelet posicionó en la esfera pública la idea de malestar y crisis en la educación (Ruiz, 2015; autor, 2019). Una movilización de alcance nacional cuyo objetivo central fue la derogación de la Ley Orgánica Constitucional de Enseñanza (LOCE), heredada de la dictadura, como marco 
legal que sustentaba la educación como un bien de mercado, exigiendo al Estado un rol más activo y menos subsidiario en relación con el funcionamiento del modelo educativo. Aquello significaba intervenir en dos ámbitos: a) en educación superior se apelaba a cuestionar el lucro y la mercantilización; b) en educación secundaria se llama a poner fin a la municipalización de la educación primaria y secundaria (Ruiz, 2015).

Ese año, cambian las coordenadas de la lucha estudiantil, gracias a la capacidad de la movilización pingüina de impactar en la opinión pública y la agenda política del gobierno con una demanda con alcance estructural «NO a la LOCE» y «el fin de la municipalización», presentando las primeras críticas profundas al modelo mercantilizado de educación (Donoso, 2013). Lo anterior, debido a una serie de factores, entre ellos, la definición estratégica por parte del mundo estudiantil de dos agendas que corrían en paralelo. La agenda corta, que se ocupaba de los temas más cotidianos, como gratuidad de la prueba de selección universitaria (PSU) o del pase escolar, el fin de la jornada escolar completa (JEC), o temas de infraestructura o alimentación, entre otros. Por otro lado, una agenda larga, cuyo foco se orientó a los problemas estructurales de la educación, en particular los de financiamiento y calidad (Donoso, 2013; Aguilera, 2011).

Junto a estas opciones estratégicas, se dieron algunos factores culturales. En primer lugar, el repertorio de movilización estudiantil se revitaliza (Donoso, 2013; Aguilera, 2011), al darle una cara más lúdica, diferenciándose de las protestas estudiantiles anteriores sobre la base de una lucha más confrontacional. El nuevo repertorio incorporó la centralidad de la ocupación de establecimientos educacionales o «tomas», que facilitaron algunas intervenciones de carácter creativo en los frontis de los liceos y en las performances callejeras, como por el uso de redes sociales, especialmente los fotologs. En las tomas y en el uso de los fotologs, el encuadre fue muy creativo, interpelando directamente a la institucionalidad política con mensajes como «La LOCE es Loser»; a la presidenta Bachelet le preguntaron «¿Estás conmigo?», emulando su eslogan de campaña; o la iconografía del pingüino rebelde presente en la mayoría de los fotologs y lienzos. Mensajes de denuncia, protesta y demandas, que reforzaron la identidad estudiantil bajo el ícono del pingüino.

Un segundo factor remite al carácter generacional de las y los jóvenes estudiantes movilizados. El uso de los fotologs permite considerarlos como parte de un período de movilización social de alcance global que utilizó las redes sociales para difundir sus mensajes, realizar sus convocatorias y estimular la participación (Juris, 2006). El uso de fotologs por parte de los estudiantes del 
Instituto Nacional, el Instituto Nacional Barros Arana o el Liceo Confederación Suiza, por nombrar algunos, le permitieron al movimiento aumentar su capacidad de visibilizar la movilización y sus demandas, al mismo tiempo de hacerla más llamativa en aspectos estéticos y comunicacionales (Aguilera, 2011), como también para contrarrestar los mensajes del gobierno condenando la movilización. La creación de otros fotologs, exclusivamente vinculados a la movilización estudiantil, como «Tómate un Liceo»o «Paro en Concepción», aumentó las potencialidades de resonancia simbólica (Tarrow, 2013) de la causa estudiantil.

Un tercer factor relevante fue la coordinación de una serie de organizaciones estudiantiles de secundaria que conformaron un cuerpo relacionado y unificado, sobre la base de un procedimiento participativo de carácter asambleario y horizontal (Donoso, 2013), fomentado principalmente por la Asamblea Coordinadora de Estudiantes Secundarios. La forma asamblearia y la horizontalidad como práctica, no fueron solo procedimientos de toma de decisión, sino que difundieron y consolidaron un estilo colectivo y una lógica de hacer política (Valenzuela Fuentes, 2015). Como expone un entrevistado:

Me acuerdo que la última asamblea en el Nacional había unas 600 personas. Entonces, yo creo que una de las cuestiones que fomentó el 2006, en el mundo estudiantil, fue la capacidad de entender que en nosotros había una responsabilidad. Esa responsabilidad no es solo de los partidos políticos, ni de las organizaciones sociales ni de las organizaciones políticas no partidistas... sino de nosotros en conversaciones tipo asamblea... Por ejemplo, me acuerdo que una de las grandes inquietudes era «icuándo hay asamblea?, ¿cuándo vamos a discutir esta cuestión?». Entonces, ese fue el principal mecanismo de organización que tuvimos. (Activista 2006, universidad privada, Santiago).

Un cuarto factor cultural, resultado de la combinación de los tres anteriores, fue el alto apoyo ciudadano logrado por la movilización en sus primeras semanas (Aguilera, 2011), en particular por la represión policial que sufrieron, junto con la implementación de un repertorio de movilización creativo y no tan violento. Sobre la base de estos factores, el año 2006 se configuró como una coyuntura polémica que fue un hito para las movilizaciones estudiantiles posteriores.

El 2008, secundarios y universitarios se movilizaron en contra de la promulgación de la Ley General de Educación (LGE), diseñada en respuesta a la revolución pingüina, aunque sin incluir las propuestas estudiantiles en su 
formulación. Contra los intereses del movimiento, el año 2009 se promulgó la LGE. Paralelamente, entre los años 2000-2010 el endeudamiento de los estudiantes universitarios aumentó, debido al uso del crédito bancario avalado por el Estado (Kremerman y Páez, 2016), al punto que, el Estado pagó a la banca la cifra de 3,1 billones de pesos por la compra de créditos CAE durante el período 2006-2017 (Fundación Sol, 2018). El 2010 asume la presidencia Sebastián Piñera, representante de la centroderecha chilena, poniendo fin a cuatro períodos presidenciales de la centroizquierda. Estos puntos definieron el escenario futuro para nuevas protestas estudiantiles.

El carácter generacional de la demanda del 2006, con el uso de nuevas tecnologías para la difusión y publicitación de su causa, el uso de la asamblea masiva como forma de conducir políticamente y la presencia de un liderazgo más horizontal revitalizaron el repertorio de movilización, lo que a su vez potencia la creación de una identificación colectiva, el movimiento estudiantil pingüino (Aguilera, 2011; Donoso, 2013). La presencia de este actor colectivo en el espacio público se mantuvo - si bien con altibajos - hasta antes del terremoto del 2010. En este actor se encarnaron los elementos político-culturales que beneficiaron la capacidad de movilización de los estudiantes en torno a un objetivo común: la crisis de la educación mercantil, de cara al escenario del 2011.

\section{LA COYUNTURA DE LA GRATUIDAD Y EL FIN AL LUCRO (2011)}

Durante gran parte del año académico del 2011 se realizaron masivas marchas, plagadas de acciones artísticas y carnavalescas; la creatividad en las formas de manifestación y la pluralidad de cursos de acción fue la tónica del período. Los universitarios, con el apoyo de los secundarios, encontraron en la protesta masiva la clave para reinstalar en el espacio público la denuncia de la crisis de la educación y demanda por la educación pública (autor, 2019). Con el correr de los meses, las manifestaciones fueron escalando en masividad, en una ola progresiva de acciones de protesta (Segovia y Gamboa, 2012). Paros y tomas de universidades y liceos en todo Chile, además de un conjunto de performances lúdicas y creativas como el «Thriller» de Michael Jackson o correr en torno al Palacio de La Moneda durante 1.800 horas, junto a acciones más tradicionales como «los banderazos» $\mathrm{y}$ «cacerolazos» e incluso la huelga de hambre definieron un repertorio diverso y complejo que caracterizó a la manifestación estudiantil del período. El siguiente testimonio sintetiza la riqueza cultural del momento: 
Nosotros participamos de todas esas cosas, del Thriller por la educación, pusimos gente ahí, porque era otra forma más lúdica de mostrar las demandas, de hacer el movimiento más transversal [...] Yo me acuerdo, inventamos un juego, la Lucrópoli de la educación, e íbamos a Plaza Italia y Plaza de Armas a jugar con la gente en la calle, y era como vivir en el mundo educacional... algo así como te endeudaste, te vas a la cárcel 10 años, o te atrasaste con la carrera. Era muy divertido, porque andábamos movilizando a la gente en las calles, era otra forma de llevar las demandas más allá de lo que muestra la prensa, era pelear contra la versión oficial. (Activista 2011, universidad pública, Santiago)

No obstante la plétora de actuaciones, el recurso más característico del repertorio fue la marcha pública, masiva y lúdica (Paredes, 2018). Bajo una coordinación nacional a través de la Confederación de Estudiantes de Chile (CONFECH) y la participación de las principales organizaciones de estudiantes secundarios, como la Asamblea Coordinadora de Estudiantes Secundarios (ACES) o la Coordinadora Nacional de Estudiantes Secundarios (CONES), la movilización funcionó bajo el formato de asambleas periódicas en el que se toman decisiones sobre diversos temas, previamente discutidos en las bases universitarias, lo que deja las influencias de la movilización pingüina (Paredes, 2019). La marcha se destacó por un desfile de colores, música, cánticos y consignas, que involucró batucadas, chinchineros, performances, expresiones artísticas, rayados, aunque no se descartó la realización de enfrentamientos y acciones violentas. Ellas lograron una masividad transversal, no recordada desde la lucha contra la dictadura, destacando además la combinación entre la tradicional forma de procesión o liturgia política, junto con el ambiente de carnaval y festivo (Ganter et al., 2017), siendo este el primer factor cultural.

Los estudiantes tomaron el protagonismo público mediante la resonancia nacional de sus manifestaciones, desde la cuales se pueden destacar otros alcances culturales. A través de la consigna «Educación Pública, Gratuita y de Calidad», la movilización consiguió importantes índices de convocatoria y una alta adhesión ciudadana a la causa por el derecho a la educación. Los estudiantes junto con defender la educación pública, consiguen inscribir en lo público la idea de «Fin al lucro», logrando que miles de chilenos solidaricen con la causa estudiantil (Paredes, 2018; Montero et al., 2017), a tal punto que la crítica al lucro se extiende a otros bienes públicos (salud y pensiones). El encuadre político del movimiento estudiantil señala la existencia de un modelo social injusto que reproduce constantemente la desigualdad, fomentado por una alianza entre el mundo político y el económico (Paredes y Otárola, 2019). El segundo factor cultural remite al encuadre realizado por el movimiento en tanto diagnóstico de 
la situación de la educación y el pronóstico de su superación: el derecho a la educación y su desmercantilización (fin al lucro).

En tercer lugar, el 2011 significó un giro ciudadano en la agenda política. Entre mayo y diciembre de ese año, el principal tema de interés nacional fueron las acciones que el movimiento estudiantil realizaba de manera pública. Desde el gobierno, en respuesta a la presión estudiantil, surgen una serie de medidas, las que fueron insuficientes para el estudiantado. A partir de ese año, la manifestación pública se convierte en una herramienta legítima de uso masivo, marcando la ruta hacia la politización de la sociedad mediante la lucha callejera (Paredes, 2018). Politización que se expande rápidamente por la sociedad chilena en los años siguientes (PNUD, 2015; Ruiz, 2015; Paredes, 2019), hacia otras problemáticas como la demanda por una nueva Constitución o el cambio del sistema de pensiones.

En cuarto lugar, se produce un fuerte efecto de legitimidad de los actores colectivos movilizados. En las calles se deja ver, durante todo ese año, una presencia colectiva que responde al nombre de estudiantes en la lucha, al tiempo que genera un efecto sobre la ciudadanía que comienza a movilizarse en apoyo a la causa estudiantil, adquiriendo un carácter activista (Paredes, 2018). Se reforzó la identificación colectiva del mundo estudiantil unificado a pesar de las diferencias internas que existían, más allá del carácter pingüino, y se generó una identificación ciudadana movilizada, la que se proyectará en los años venideros en otras expresiones colectivas como el NO+AFP.

En quinto lugar, la intensidad de la movilización es atacada violentamente por el gobierno mediante una fuerte represión policial. Ello implicó la recuperación histórica de prácticas de protesta como el cacerolazo (Ortiz, 2019), mediante un ejercicio de memoria para denunciar el abuso y la represión policial. Esto estrechó la relación entre estudiantes en la lucha y ciudadanía movilizada, pues permitió un ejercicio de liberación emocional (Flam, 2005), donde el difuso malestar social dio paso a una clara indignación por el abuso policial y luego al orgullo de compartir un compromiso con la movilización. Tal cambio en el registro emocional colectivo es una de las secuelas más relevantes del período, porque reforzará la legitimidad pública de la protesta callejera y de los actores colectivos, pues la indignación y el orgullo serán emociones movilizadoras (Flam, 2005) en otras movilizaciones y no solo las estudiantiles. Al punto que se habló de la generación sin miedo, para referir al actor movilizado durante el ciclo 2006-2011 (Sandoval y Carvallo, 2019). 
La constitución del actor colectivo se amplificó incluyendo a otros grupos sociales y a sectores de la ciudadanía, aunque siguió siendo el movimiento estudiantil por la educación pública, universitaria y secundaria el eje identitario más relevante (Paredes, 2018). Ella se reforzó por la elaboración de un marco de movilización sustentado en el derecho a la educación y el fin al lucro, definiendo una base emocional y moral que dotó de legitimidad a la causa pública y la proyectó por más de cinco años (Paredes y Otárola, 2019).

En los años posteriores, el protagonismo contencioso fue compartido entre universidades estatales, universidades privadas y estudiantes secundarios, que continuaron realizando marchas masivas de carácter lúdico, con el fin de conseguir el apoyo ciudadano para lograr el derecho a la educación y su desmercantilización. Sin embargo, el movimiento estudiantil compartirá el escenario público con otras movilizaciones sociales, lo que le restó relevancia y centralidad. No obstante, una de las secuelas al combinar los vectores señalados anteriormente es el cambio en el régimen de visibilidad (Aguilera, 2011) de las manifestaciones del actor estudiantil y su legitimidad, que continuó teniendo efectos a futuro.

\section{La COYUntura Feminista (2018)}

El movimiento estudiantil retoma centralidad el 2018, pero ahora con una característica distintiva: su rostro femenino. Ya no es la mercantilización o el derecho a la educación su leitmotiv, sino la denuncia del conjunto de situaciones de abuso e injusticia que viven las mujeres en los espacios educativos, la crítica al patriarcado como forma sociocultural dominante y a la forma en que se distribuye el poder entre mujeres y hombres en la sociedad (Motta et al., 2020; De Fina Gonzalez y Figueroa, 2019; Reyes-Housholder y Roque, 2019).

El mayo feminista (Zerán, 2018; Reyes-Housholder y Roque, 2019) es fruto de un largo proceso de luchas que se arrastra desde la dictadura y se mantiene durante la democracia (De Fina Gonzalez y Figueroa, 2019; Lamadrid y Navarrete, 2019), que ve en ese año su punto más álgido. El repertorio de actuación siguió una línea común respecto al 2006 y 2011, en tanto nuevamente se recurre a las manifestaciones festivas masivas y performances públicas de resonancia político-cultural. Uno de los recursos distintivos de las manifestaciones feministas fue el uso del cuerpo y su liberación, por ejemplo, marchar con el torso desnudo y pintado, para desafiar las normas y el poder de la 
cultura masculina (Reyes-Housholder y Roque, 2019), alterando el régimen de visibilidad de un espacio público de preferencia masculino. La fuerza feminista del movimiento estudiantil, durante ese año, movilizó a más de 150.000 manifestantes, solo en Santiago. Por otro lado, se realizaron más de veinte tomas de campus universitarios en todo el país (Motta et al., 2020), y también un conjunto de liceos secundarios fueron tomados en apoyo a la movilización, mostrando la continuidad del repertorio de protesta.

En términos de encuadre, son dos las demandas que operaron como marco de la movilización. Por un lado, en el plano educativo se demandó incorporar en el debate la educación no sexista. En un plano más sociopolítico, la denuncia y crítica apuntó al patriarcado como lógica cultural y estructura social, con sus variadas formas de violencias e injusticias (De Fina Gonzalez y Figueroa, 2019). Este marco tuvo una resonancia simbólica muy fuerte en la sociedad, pues encontró eco en las mujeres en general y no solo en las estudiantes, pero también en disidencias, minorías e incluso en el mundo masculino. Nuevamente, se produce una forma de configuración colectiva estudiantil, ahora en clave feminista, pero también una configuración colectiva que desbordó con creces los espacios educativos.

En tercer lugar, la forma de participación y deliberación se dio en espacios feministas autoconvocados, donde la práctica horizontal y asamblearia se replicó durante el mayo feminista (Reyes-Housholder y Roque, 2019). Al interior de universidades y liceos se realizaron asambleas, talleres, espacios de conversación o círculos feministas, que a través de un ejercicio deliberativo fueron compartiendo y difundiendo un conjunto de experiencias compartidas y situaciones comunes vivenciadas por las mujeres al interior de las instituciones educativas (Motta et al., 2020).

Tales expresiones de deliberación se ampliaron más allá del estamento estudiantil, para incluir a docentes y profesoras, funcionarias diversas, administrativas y directivas, que definieron formas de intervención en sus organizaciones - mediante la creación de protocolos, reglamentos y unidades de género- - También repercutieron en la cultura organizacional, al legitimar en sus espacios los feminismos como prácticas y perspectivas, pero también en el ámbito de las subjetividades — no solo las feministas-, pues en varios espacios se dio una reflexión colectiva por parte de los estudiantes masculinos en relación con sus privilegios y la reproducción de prácticas machistas. Un logro significativo de la movilización en los espacios académicos fue la incorporación del lenguaje inclusivo en ellos (Reyes-Housholder y Roque, 2019). Esa 
resonancia alcanzó también el espacio público mediante las masivas manifestaciones públicas realizadas, posicionando la denuncia feminista contra los abusos de la cultura machista al centro de la sociedad chilena.

En términos de sociabilidad y vínculos sociales, el 2018 situó en la escena pública — pues ya existía en espacios privados — un tipo de fraternidad feminista y solidaridad entre mujeres: la sororidad. Ella pasó de un recurso práctico y relacional de las mujeres en su cotidianeidad, para transformarse en un recurso emocional, hermenéutico y pedagógico, para hacer sentido y enseñar al conjunto de la sociedad, la forma colaborativa con la que las mujeres han podido sobrevivir en el mundo de la cultura machista. Por ejemplo:

Yo estoy sumamente orgullosa, y he logrado ver el proceso que implicó todo eso. Creo que es mucho más probable generar un cambio más radical en política y en la sociedad, por el movimiento feminista que por el movimiento estudiantil [porque] el movimiento feminista nos involucra a todas, da lo mismo de dónde vengas, tú eres oprimida por el patriarcado, más o menos, pero estamos todas en las mismas. (Activista 2018, universidad pública, Santiago)

Todo lo anterior consolidó la presencia del feminismo en lo público, ya sea en clave estudiantil, social o política, como la agencia colectiva del año 2018. Fue tanta la fuerza del movimiento que su resonancia se mantuvo por un tiempo, por ejemplo, se realizó a fines del 2018 un masivo encuentro feminista en la Universidad de Santiago de Chile, con la tarea de definir la agenda para el año siguiente, cuya traducción fue la exitosa jornada del 8 de Marzo del 2019, cuando el territorio nacional se volvió feminista gracias a una de las marchas más masivas de los últimos años.

Aunque la identificación pública de la movilización del 2018 varió en relación con las anteriores, al anteponer la etiqueta feminista antes de la estudiantil, nuevamente se elaboró, a pesar de sus diferencias internas, una actoría colectiva afiatada (Motta et al., 2020), que sobre la base de performances llamativas y un repertorio de actuación lúdico y contestatario, consolidó formas prácticas y estilos de sociabilidad horizontal, junto con un encuadre crítico y emocional, de gran resonancia pública. Lo que permite sostener la hipótesis de la continuidad de factores culturales en las tres coyunturas. 


\section{DisCuSIÓN: CONSECUENCIAS CULTURALES PARA LA COYUNTURA CRÍTICA DEL OCTUBRE CHILENO}

«Evadir, no pagar, otra forma de luchar», gritaron los estudiantes días previos al 18-O, invitación que posteriormente derivó en una gran revuelta popular de alcances aún por ponderar. Hemos presentado tres coyunturas polémicas a partir de diferentes expresiones del movimiento estudiantil del siglo XXI. Siguiendo sus huellas, sugerimos cuatro alcances político-culturales que moldean el paisaje cultural de la revuelta popular chilena.

En primer lugar, planteamos que el trayecto analizado fomentó un proceso de subjetivación colectiva vía politización (Paredes, 2019), por el cual los actores sociales abandonaron su pasividad previa para definirse a partir de otra clave, más política y contenciosa. En el mundo estudiantil se constituye la figura colectiva del estudiante en la lucha, ya sea por el cambio de las reglas del juego (fin de la LOCE), por el fin de la mercantilización y la instauración de ella como un derecho garantizado por el Estado, o por la educación no sexista y el fin de los abusos y privilegios machistas en las instituciones educativas. En cada una de ellas, el estudiantado fue un sujeto político y no un mero estudiante. Tal proceso de subjetivación vía politización alcanzó también a la ciudadanía, en especial durante 2011 y 2018, generando un activismo ciudadano en cada coyuntura, no restringido a ellas, pues operó con un efecto de contagio para la conformación de otros actores colectivos con sus propias luchas. El primer registro, entonces, es el de una subjetivación colectiva vía politización, que algunos trabajos han leído en clave generacional (Sandoval y Carvallo, 2019; Santibáñez y Ganter, 2016).

Tal contagio permite reconocer un segundo nivel, asociado a las acciones y estilos colectivos en cada una de las coyunturas. En la dimensión interna a los procesos de movilización, practicaron formas de participación deliberativas y decisión horizontal, bajo un estilo asambleario (Valenzuela Fuentes, 2015). En su dimensión externa, el uso frecuente de la manifestación pública y la protesta, junto con la legitimidad social que fue adquiriendo, mediante un fuerte respaldo público y ciudadano (Paredes y Araya, 2020). En especial, las marchas carnavalescas o formas de actuación lúdica como los flashmobs, las ocupaciones no violentas de edificios públicos y privados, diferentes tipos de performance de protesta, entre otras (Paredes, 2018). Simultáneamente, se recuperaron modalidades históricas de protesta, como los cacerolazos, que potenciaron el apoyo a la causa estudiantil en los territorios y las plazas. Con ello definieron al espacio público como el lugar para exponer su demanda, mediante diferentes 
formas de manifestación pública en cada coyuntura, difundiendo un estado de ánimo común y un sentimiento de injusticia colectivo (Paredes y Otárola, 2019), que se replicó durante el largo octubre chileno.

Un tercer registro, entonces, remite a la elaboración de una batería emocional (Jasper, 2016) propia de las coyunturas movilizadas, sustentadas en emociones morales asentadas. El par indignación/orgullo fueron las emociones movilizadoras (Flam, 2005) que han solventado la acción colectiva. Por otra parte, las coyunturas polémicas han actuado como procesos de liberación emocional (Flam, 2005) para los actores colectivos y la ciudadanía individualizada. El paso del malestar difuso a la indignación concreta, el $2011 \mathrm{y}$ 2018, ha cambiado las reglas del sentir social (Hochschild, 1979), donde se dan formas de experiencia emocional que puedo compartir con otros en el espacio público, por ejemplo, las emociones morales de la dignidad y la esperanza como sostén de la continuidad del largo Octubre de 2019. De tal manera, los registros emocionales y la modificación de las reglas del sentir (Gravante, 2020) son una de las consecuencias más significativas de las protestas estudiantiles, especialmente al fomentar solidaridad y sororidad entre activistas y ciudadanos.

La capacidad de instalar un vocabulario público es la cuarta contribución de alcance cultural. Toda movilización genera su lenguaje de protesta (Tarrow, 2013), como forma de inscripción pública de ideas y conceptos. Las diversas coyunturas polémicas fomentadas por la acción colectiva estudiantil han inscrito públicamente su vocabulario de injusticia como de propuestas, logrando tanto resonancia simbólica y modularidad (Tarrow, 2013). Ya sea mediante el vocabulario afirmativo de los derechos sociales y del reconocimiento, encarnado en la educación gratuita o en la igualdad hacia las mujeres, o en el lenguaje crítico del fin de la mercantilización o de la violencia hacia las mujeres, se ha generado todo un trasfondo de sentido cultural (Alexander, 2003), que favoreció la denuncia de múltiples formas de injusticia y los reclamos de justicia que sostuvieron la revuelta popular del 2019.

Tal vocabulario, al combinarse con las prácticas y las emociones morales, sugiere la inscripción pública de una economía moral (Fassin, 2009), contraria a la forma neoliberal, basada en los derechos sociales y el reconocimiento de la desigualdad social, que sostuvo la movilización. Entendemos la economía moral como una modalidad de producción, distribución, circulación y uso de emociones morales, valores, normas y obligaciones (Fassin, 2009) en la vida social. Sugerimos que se ha conformado una economía moral popular o ciudadana de la 
justicia social, que descansa en la idea de los derechos sociales, mediante la tarea de la redistribución y del reconocimiento. Una economía moral contraria a los abusos y que se configura a partir del reclamo por el derecho a la educación o de los derechos de las mujeres. Entonces, las movilizaciones estudiantiles han desplegado como efecto político-cultural una economía moral de la justicia opuesta a la mercantilización de la vida social, que fomenta múltiples situaciones de abuso e injusticia.

Señalamos que los factores culturales identificados permiten definir cada coyuntura particular con un alcance polémico, puesto que exponen una crítica social consistente a la desigualdad y a las violencias por ella provocadas, limitada a la educación o al género, pero sin alcanzar las bases mismas del modelo social. Sin embargo, sugerimos que tales consecuencias, en su articulación, crean condiciones político-culturales favorables para la emergencia del largo octubre chileno, al poner en relación dimensiones agenciales y estructurales de manera generalizada, a partir de la radicalización y acumulación de la experiencia en las luchas anteriores. Las anteriores cuatro consecuencias, pueden organizarse en tres claves problemáticas que definen la coyuntura como totalidad epistémica (Grossberg, 2010), de gran calado político-cultural: a) el nivel subjetivo (subjetivación colectiva vía politización); b) nivel de la resistencia y la agencia (manifestación pública/estilo asambleario y cambio en las reglas del sentir); c) nivel ideológico-discursivo (vocabulario de derechos y economía moral). Estas tres problemáticas en su articulación contextual, vincularon la dimensión de los actores, la acción y la estructura política-ideológica, favoreciendo que el acontecimiento de octubre se convirtiera en una coyuntura crítica, pues cuestionó las bases mismas del modelo debido a la resonancia de sus demandas con el trasfondo cultural propiciado, entre otras razones, por las movilizaciones estudiantiles de los últimos quince años.

La educación nuevamente marcó el paso, con una intervención realizada en el Metro de Santiago a inicios de octubre 2020, para la conmemoración a un año del 18-O. En la ocasión, estudiantes desplegaron un lienzo con la leyenda «Lo que empiezan lxs secundarixs, lo termina el pueblo organizado», confirmando parte del argumento del texto, relativo a la generalización de la experiencia político-cultural a partir de la protesta. El mundo estudiantil ha demostrado que cada coyuntura polémica (2006, 2011 y 2018), marcada por protestas sociales y manifestaciones públicas, fue un laboratorio de experiencia colectiva que, en su generalización y acumulación, generó ciertas condiciones 
político-culturales para la emergencia del 18-O y su transformación en una revuelta popular de varios meses, durante el 2019.

Varios de los puntos expuestos son reconocidos, aunque de otra forma y en otro lenguaje, por el Informe PNUD (2019) como elementos que definen un cambio cultural en la ciudadanía, antes de 2019. Aunque los efectos mencionados no permiten definir el timing de la revuelta, sí permiten hipotetizar razones de su ocurrencia y sustentar parte de su persistencia en el tiempo en factores culturales, como por ejemplo la presencia en años anteriores de actores colectivos politizados en el espacio público, que fomentan un vocabulario de derechos, una economía moral y un cambio en las reglas del sentir público, sobre la base de la protesta como práctica legitimada socialmente. Lo anterior genera condiciones favorables para dar sentido al cambio del paisaje político-cultural del Chile reciente, orientado a la democratización de la sociedad chilena, donde el fin de la Constitución de 1980 es solo el comienzo.

RECIBIDO: 28 DE OCTUBRE DE 2020

ACEPTADO: 21 DE DICIEMBRE DE 2020

\section{BIBLIOGRAFÍA}

Aguilera, O. (2011). Acontecimiento y acción colectiva juvenil. El antes, durante y después de la rebelión de los estudiantes secundarios chilenos en el 2006. Propuesta Educativa, 1(35), 11-26.

. (2012). Repertorios y ciclos de movilización juvenil en Chile (20002012). Utopía y Praxis Latinoamericana, 17(57), 101-108.

Alexander, J. (2003). The Meaning of Social Life. A Cultural Sociology. Oxford: Oxford University Press.

Amenta, E. y Polletta, F. (2019). The Cultural Impacts of Social Movements. Annual Review of Sociology, 45, 279-299. http://dx.doi.org/10.1146/annurev-soc-073018-022342

Angelcos, N. y PÉREZ, M. (2017). De la «desaparición» a la reemergencia: Continuidades y rupturas del movimiento de pobladores en Chile. Latin American Research Review, 52(1), 94-109. DOI: http://doi.org/10.25222/larr.39

AssaÉl, J., Cornejo, R., GonzÁlez, J., Redondo, J., SÁnchez, R. y Sobarzo, 
M. (2011). La empresa educativa Chilena. Educação \& Sociedade, 32(115), 305-322. $\quad \mathrm{https} / / / \mathrm{dx} . d o i . o r g / 10.1590 / \mathrm{S} 0101-$ 73302011000200004

AtriA, F. (2014). Derechos Sociales y Educación: El Nuevo Paradigma de lo Público. Santiago de Chile: Lom Editores.

AVENDAÑO, O. (2014). Fracturas y representación política en el movimiento estudiantil: Chile 2011. Última Década, 22(41), 41-68.

BÁEZ, F. (2017). Diseño institucional y Neoliberalismo. El modelo chileno como resultado del quiebre unilateral del contrato social. Papers, 102/3, 449476. http://dx.doi.org/10.5565/rev/papers.2299

BelleI, C. (2015). El gran experimento. Mercado y privatización de la educación chilena. Santiago de Chile: Lom Editores.

Bellei, C., Cabalín, C. y Orellana, V. (2014). The 2011 Chilean student movement against neoliberal educational policies. Studies in Higher Education, 39(3), 426-440. https://doi.org/10.1080/03075079.2014.896179

Berroeta, H. y Sandoval, J. (2014). Protestas, participación y educación pública: discursos sobre lo público en las movilizaciones estudiantiles en Chile. Educar em Revista, 53, 19-38. Doi: 10.1590/0104-4060.36578

Bosi, L., GiUgni, M. y UBA, K. (2016). The Consequences of Social Movements. Cambridge: Cambridge University Press.

CABALÍn, C. (2014). Online and Mobilized Students: The Use of Facebook in the Chilean Student Protests. Comunicar 43, 25-33. https://doi.org/10.3916/C43-2014-02

CÁRdenAs, C. (2016). El movimiento estudiantil chileno (2006-2016) y el uso de la web social: nuevos repertorios de acción e interacción comunicativa. Última Década, 24(45), 93-116.

CEFAÏ, D. (2011). Diez propuestas para el estudio de las movilizaciones colectivas. Contexto de experiencias y problemas públicos. Revista de Sociología de la Universidad de Chile, (26), 137-166. DOI: 10.5354/0719-529X.2011.27491

Cuevas, H. y Paredes, J. P. (2012). La ciencia política y el campo de los estudios cualitativos interpretativos de la política. Revista Pléyade, 10, 114.

De Fina Gonzalez, D. y FigueroA, F. (2019). Nuevos «campos de acción política» feminista: Una mirada a las recientes movilizaciones en Chile. Revista Punto Género, (11), 51-72. DOI:10.5354/0719-0417.2019.53880

Delamaza, G., Maillet, A. y Martínez-Neira, C. (2017). SocioTerritorial Conflicts in Chile: Configuration and Politicization (2005-2014). 
European Review of Latin American and Caribbean Studies, 104, 23-46.

Donoso, S. (2013). Dynamics of Change in Chile: Explaining the Emergence of the 2006 Pingüino Movement. Journal of Latin American Studies, 45(1), 1-29. https://doi.org/10.1017/S0022216X12001228

EARL, J. (2004). The Cultural Consequences of Social Movements. En D. SNOw, S. Soule y H. KRIESI (Eds.), The Blackwell Companion to Social Movements (pp. 508-530). Oxford: Blackwell.

Fassin, D. (2009). Moral Economies Revisited. Annales. Histoire, Sciences sociales, 64(6), 1237-1266.

Flam, H. (2005). Emotions' Map. A Research agenda. En H. Flam y D. King (Eds.), Emotions and Social Movements (pp. 19-40). Nueva York: Routledge.

Fleet, N. y Guzmán-Concha, C. (2017). Mass Higher Education and the 2011 Student Movement in Chile: Material and Ideological Implications. Bulletin of Latin American Research, 36(2), 160-176. https://doi.org/10.1111/blar.12471

FLEET, N. (2011). Movimiento estudiantil y transformaciones sociales en Chile: una perspectiva sociológica. Polis, 10(30), 99-116. http://dx.doi.org/10.4067/S0718-65682011000300005

FUNDACIÓN SOL. (2018). CAE 2018: Endeudar para gobernar y mercantilizar. Recuperado de: http:/www.fundacionsol.cl/estudios/cae2018/

GANTER, R., VERGARA, C. y FuICA, I. (2017). Caleidoscópolis: signos de cambio en los repertorios de protesta callejera en la ciudad de Concepción-Chile. Universum, 32(2), 81-105. https://dx.doi.org/10.4067/S071823762017000200081

GÁRATE, M. (2012). La revolución capitalista de Chile (1973-2003). Santiago de Chile: Ediciones Universidad Alberto Hurtado.

GiUgni, M. (2008). Political, biographical, and cultural consequences of social movements. Sociology Compass, 2, 1582-1600.

GRAVANTE, T. (2020). Emociones y reglas del sentir como impactos culturales de los movimientos sociales. Interdisciplina, 8(22), 157-179. Doi: http://dx.doi.org/10.22201/ceiich.24485705e.2020.22.76423

Grossberg, L. (2010). Cultural Studies in the Future Tense. Durham: Duke University Press.

GuSFIELD, J. (1981). The culture of public problems: the drinking driving problem and the production of simbolic order. Chicago: University of Chicago Press.

Hall, S. (2016). Cultural Studies 1983. A Theoretical History. Durham: Duke 


\section{University Press}

Hatibovic, F., Sandoval, J. y Cárdenas, M. (2012). Posiciones de sujeto y acción política universitaria: análisis de discurso de estudiantes de universidades de la región de Valparaíso. Última Década 37.

HeISS, C. (2020). ¿Por qué necesitamos una Nueva Constitución? Santiago de Chile: Aguilar.

HochschiLD, A. R. (1979). Emotion work, feeling rules, and social structure. American Journal of Sociology, 85, 551-75.

JASPER, J. (1997). The art of moral protest: cultural, biography, and creativity in social movements. Chicago: University of Chicago Press.

. (2016). Protesto. Uma introducao aos movimentos sociais. Río de Janeiro: Zahar.

Johnston, H. (2009). Protest Cultures: Performance, Artifacts, and Ideations. En H. Johnston, Culture, Social Movements, and Protest (pp. 3-29). Farnham: Ashgate.

Julián, D. (2018). Características organizacionales del sindicalismo en Chile. Investigación-acción en sindicatos de la Región de la Araucanía. Revista mexicana de ciencias políticas y sociales, 63(232), 269-300.

JURIS, J. (2006). Movimientos sociales en red: Movimientos globales por una justicia global. En M. CASTELLS (Ed.), La sociedad red: Una visión global (pp. 415-439). Madrid: Alianza.

KREMERMAN, M. y PAÉZ, A. (2016). Endeudar para gobernar y mercantilizar. El caso del CAE. Santiago: Fundación Sol. Disponible en: http://www.fundacionsol.cl/wp-content/uploads/2016/04/Estudio-CAE2016.pdf

LABARCA, J. T. (2016). El «ciclo corto» del movimiento estudiantil chileno: ¿conflicto sectorial o cuestionamiento sistémico? Revista mexicana de sociología, 78(4), 605-632.

LAmadrid, S. y NAVArRete, A. (2019). Cronología del movimiento feminista en Chile 2006-2016. Revista Estudos Feministas, 27(3), e54709. Epub October 21. https://doi.org/10.1590/1806-9584-2019v27n354709

MadariagA, A. (2019). La continuidad del neoliberalismo en Chile: ideas, instituciones e intereses. Revista de Estudos e Pesquisas sobre as Américas, 13(2), 81-113. DOI: https://doi.org/10.21057/10.21057/repamv13n2.2019.23217

Mella, M., Ríos, H. y RiverA, R. (2016). Condiciones orgánicas y correlaciones de fuerza del movimiento estudiantil chileno: Una aproximación desde la Confech (2011-2015). Izquierdas, 27, 134-160. 
Montero, V. (2018). Movimientos sociales y consecuencias político institucionales. Del movimiento universitario 2011 a la Reforma en educación superior en Chile. Persona y Sociedad, 32(2), 46-88.

Montero, V., MuÑoz C. y PiCAZO, M. (2017). Estrategias y recursos empleados por el movimiento estudiantil en el 2011. Universum, 32(1), 137-157.

MonTONI, A. (2019). Juventud militante y radicalización política: las emociones durante la contestación estudiantil chilena. Desafíos, 31(2), 169-196. Doi:http://dx.doi.org/10.12804/revistas.urosario.edu.co/desafios/a.7305.

Motta, S. C., Bermúdez Gómez, N. L., Valenzuela Fuentes, K. y Dixon, E. S. (2020). Student Movements in Latin America: Decolonizing and Feminizing Education and Life. Oxford Research Encyclopedia of Politics. https://doi.org/10.1093/acrefore/9780190228637.013.1721

Orellana, V., Canales, M., Bellei, C. y Guajardo, F. (2019). Individuación y mercado educacional en chile. Revista Brasileira de Politica e Administração da Educação, 35(1), 141-157. Epub 06 de dezembro de 2019. https://dx.doi.org/10.21573/volln12019.89879

OrTIZ, N. (2019). Cacerolazo: emociones y memorias en el movimiento estudiantil 2011. Polis Revista Latinoamericana, 53, 66-77. Doi: http://dx.doi.org/10.32735/S0718-6568/2019-N53-1385

PARedes, J. P. (2019). De la Revolución pingüina a la Arena de la gratuidad. Balance de 10 años de luchas estudiantiles en Chile (2007-2017). En R. GARcía DíEz y G. BeTANCuR (Eds.), Movimientos sociales, acción colectiva y cambio social en perspectiva. Continuidades y cambios en el estudio de los movimientos sociales (pp. 131-145). Barcelona: Fundación Betiko/FES.

. (2018). En la calle y sin permiso, yo me educo y organizo. La manifestación por la Educación Pública como forma de politización de la juventud chilena. En R. TORRES (Ed.), Juventud, espacios públicos y participación en Chile y América Latina (pp. 31-55). Santiago de Chile: UCEN/RIL.

Paredes, J. P. y Araya, C. (2020). La educación chilena, ¿no se vende? Movilización estudiantil y la configuración del problema público universitario. Polis, 19(57), 331-359. https://dx.doi.org/10.32735/s07186568/2020-n57-1573

Paredes, J. P. y OtÁrola, C. (2019). El grito ciudadano en la lucha por el derecho a la educación: El marco moral y emocional de la movilización estudiantil en Chile (2011-2013). Sociológica, 98(34), 253-288.

Paredes, J. P., Ortiz, N. y Araya, C. (2018). Conflicto social y subjetivación política: performance, militancias y memoria en la movilización estudiantil post 2011. Persona y Sociedad, 32(2), 122-149. 
Penaglia, F. y Mejías, S. (2019). El conflicto estudiantil chileno y sus efectos políticos. Polis, 15(2), 7-3.

PINEDA, C. (2012). Dominación y emancipación en el pueblo mapuche. El pensamiento de la Coordinadora Arauco Malleco. Subhistoria, 4, 43-72.

PNUD, CHILE. (2015). Los tiempos de la politización. Santiago de Chile: PNUDChile. Recuperado de: http://www.cl.undp.org/content/dam/chile/docs/desarrollohumano/Infor me\%202015.pdf

. (2016). Desiguales. Orígenes, cambios y desafios de la brecha social en Chile. Santiago de Chile: PNUD, Chile. Recuperado de: file:///C:/Users/pared/Downloads/undp_cl_pobreza-Libro-

DESIGUALES-final.pdf

. (2019). Diez años de auditoría a la Democracia en Chile: antes del estallido. Santiago de Chile: PNUD, Chile. Recuperado de: https:/www.cl.undp.org/content/chile/es/home/library/diez-anos-deauditoria-a-la-democracia--antes-del-estallido.html

Polletta, F. y JASPER, J. (2001). Collective identity and social movements. Annual Review of Sociology, 27, 283-305.

Reyes-Housholder, C. y Roque, B. (2019). Chile 2018: desafíos al poder de género desde la calle hasta La Moneda. Revista de ciencia política, 39(2), 191-216. https://dx.doi.org/10.4067/S0718-090X2019000200191

RozAS, J. y MAILlet, A. (2019). Entre marchas, plebiscitos e iniciativas de ley: innovación en el repertorio de estrategias del movimiento No Más AFP en Chile (2014-2018). Izquierdas 48, 1-22.

ROVIRA, C. (2007). Chile: transición pactada y débil autodeterminación colectiva de la sociedad. Revista Mexicana de Sociología, 69(2), 343-372.

RuIZ, C. (2015). De nuevo la sociedad. Santiago de Chile: Lom Ediciones.

SANDOVAl, J. y CARVAllo, V. (2019). Una generación «sin miedo»: análisis de discurso de jóvenes protagonistas del movimiento estudiantil chileno. Última Década, 27(51), 225-257.

SANTIBÁÑEZ, P. y GANTER, R. (2016). Representaciones sociales de lo político: Convergencias y divergencias del relato generacional en el gran Concepción. Última Década, 24(44), 39-70.

Segovia, C. y GamboA, R. (2012). Chile el año que salimos a la calle. Revista de Ciencia Política, 32(1), 65-85. https://dx.doi.org/10.4067/S0718090X2012000100004

Silva, E. (2009). Challenging Neoliberalism in Latin American. Nueva York: Cambridge UP. 
Somma, N. (2012). The Chilean student movement of 2011-2012: challenging the marketization of education. Interface, 4(2), 296-309.

SpIllman, L. (2020). What is Cultural Sociology? Cambridge: Polity.

SwIDLER, A. (1986). Culture in action: Symbols and strategies. American Sociological Review, 51, 273-286.

TARrow, S. (2013). The Language of Contention: Revolutions in Words, 16882012. Cambridge University Press.

Tricot, T. (2012). Movimiento de estudiantes en Chile: Repertorios de acción colectiva ¿algo nuevo? Revista F@ro, 15, 7-15.

VAN DYKe, N. y TAYLOR, V. (2018). The cultural outcomes of social movements. En D. SnOw, S. Soule, H. Kriesi y H. MCCAMmON (Eds.), The Wiley blackwell companion to social movements (pp. 482-498). Oxford: Blackwell.

VAlenzuela Fuentes, K. (2015). La primavera de Chile: autonomía y antagonismo en el movimiento estudiantil. En M. Modonesi (Comp.), Movimientos subalternos, antagonistas y autónomos en México y América Latina (pp. 181-198). Ciudad de México: UNAM.

Undurraga, T. (2014). Divergencias. Trayectorias del neoliberalismo en Argentina y Chile. Santiago de Chile: Ediciones UDP.

URZÚA, S. (2015). ¿Cómo marchan los jóvenes en el Chile de postdictadura? Algunas notas acerca de la apropiación del espacio público y el uso del cuerpo. Última Década, 42.

Williams, R. (2003). La larga revolución. Buenos Aires: Nueva Visión.

ZERÁN, F. (Ed.) (2018). El mayo feminista chileno. La rebelión contra el patriarcado. Santiago de Chile: Lom Editores. 